\title{
The Distribution of Vasopressin- or Oxytocin-Neurons Project- ing to the Posterior Pituitary as Revealed by a Combination of Retrograde Transport of Horseradish Peroxidase and Immunohistochemistry
}

\author{
Yutaka Taniguchi, Masaru Yoshida, Koichi Ishikawa, Mitsuo SuzUKI and Kazumasa Kurosumi \\ Departments of Morphology and Physiology, Institute of Endocrinology, Gunma University, Maebashi, Japan
}

Received October 27, 1987

Summary. The distribution of vasopressin (AVP)-containing or oxytocin (OXT)-containing neurons in the rat hypothalamus which project to the posterior pituitary was revealed by the combination of retrograde tracing of horseradish peroxidase (HRP) and immunohistochemistry. The majority of magnocellular neurons labeled with HRP were located in some of the hypothalamic nuclei, including the supraoptic nucleus and paraventricular nucleus. Many of these neurons were also immunostained by anti-AVP or anti-OXT. On the other hand, some of the immunostained neurons were not labeled with HRP in the dorso-medial and the most caudal parts of the paraventricular nucleus. These data confirmed previous reports concerning the distribution of AVP-or OXT-neurons projecting to the posterior pituitary, as a more direct visualization of both the neuropeptides and the retrogradely transported $H R P$ in the same tissue section was attained. In addition, some of the HRP-labeled perikarya which seemed to have direct contact with the ventricular lumen were occasionally seen; its functional significance is discussed.

There have been several reports concerning the efferent fiber projection to the posterior pituitary from the hypothalamic magnocellular neurosecretory nuclei of the rat (PETERSON, 1966; SCHERLOCK et al., 1975; HANCOCK, 1976; SAPER et al., 1976; HOSOYA and MATSUShita, 1979; ArMSTRONG et al., 1980; Kelly and SWANSON, 1980; SWANSON and KUYPERS, 1980; SWANSON et al., 1980; SOFRONIEW and SCHRELl, 1980,
1981; SAWCHENKO and SWANSON, 1982), quail (MIKAMI et al., 1979) and squirrel monkey and cat (SAPER et al., 1976). Concurrently, many reports on immunohistochemical studies concerning the distribution of AVP- or OXT-containing neurons have been published (PETER SON 1966; ARMSTRONG et al., 1980; RHODES et al., 1981). The results of these studies indicated that the anterior and lateral magnocellular regions of the paraventricular nucleus of the rat project to the posterior pituitary, while the dorso-medial and posterior regions of this nucleus project to the medulla oblongata or spinal cord. The projections to the medulla or spinal cord from some of the AVP-or OXT-containing magnocellular neurons were revealed by SOFRONIEW and SCHRELl (1981), with SAWCHENKO and SWANSON (1982) using the double staining method which revealed both the marker of the retrograde transport and the neuropeptides. However, there is yet no report that has clarified the distribution of the AVP- or OXT. containing neurons which project to the posterior pituitary using the combination of HRP study and immunohistochemistry applied to the same tissue sections. We have successfully applied this technique (WAINER and RYE, 1984 ; BEITZ et al., 1986) to the rat posterior pituitary; the results obtained are, as a whole, consistent with previous papers, but also include some new findings. To our knowledge this is the first report of a double staining study applied to the posterior pituitary. 


\section{MATERIALS AND METHODS}

\section{Tissue preparation}

Adult male rats of the Wistar strain (BW 250-350 g) were used throughout the study. The animals were anesthetized with an intraperitoneal injection of Nembutal $(50 \mathrm{mg} / \mathrm{kg} \mathrm{BW})$ and fixed on a board. The pituitary was exposed by the parapharyngeal approach (SWANSON and KUYPERS, 1980) and $0.3 \mu 1$ of 10\% HRP (grade I-C, Toyobo Co. Osaka, Japan) dissolved in saline was injected through a glass micropipette (ca. $50 \mu \mathrm{m}$ in external diameter) which was connected to a $10 \mu 1$ microsyringe. The plunger of the syringe was driven by an oil manipulator. On the next day, colchicine (100 $\mu \mathrm{g}$ in $20 \mu \mathrm{l}$ saline) was injected into the lateral ventricle; the animals were sacrificed, under Nembutal anesthesia, on the next day of colchicine injection by the cardiac perfusion of Zamboni's fixative (STEFAnini et al., 1967). The brains were removed, trimmed, and stored at $4^{\circ} \mathrm{C}$ in the fixative solution with $30 \%$ sucrose dissolved in it. The following day, $30 \mu \mathrm{m}$ frontal sections were cut on a cryostat and every fourth section was collected for subsequent steps.

\section{Detection of HRP and immunocytochemical staining}

The retrogradely transported HRP was detected by the method by ADAMS (1977) with some modifications. First, the sections were washed with PBS several times. They were then incubated in the following solution for $3-5 \mathrm{~min}$ at room temperature: $0.02 \%$ of $3-3$ ' diaminobenzidine (DAB), $0.02 \%$ of cobalt chloride, $0.005 \%$ of hydrogen peroxide in $1 / 15 \mathrm{M}$ phosphate buffer ( $\mathrm{pH}$ 7.4). The sections were washed thoroughly with PBS and then immunohistochemical staining was carried out using the unlabeled antibody method: $1 \%$ normal goat serum in TBS (PBS with $0.3 \%$ Triton X100) for $2 \mathrm{~h}$, primary antiserum (either anti-AVP 1: 2000 or anti-OXT 1:2000) for 2 days at $4^{\circ} \mathrm{C}$, TBS washing, goat anti-rabbit IgG $1: 200$ for $2 \mathrm{~h}$, TBS washing, PAP complex (Dakopatts, Denmark) 1:200 for $2 \mathrm{~h}$, TBS washing, $\mathrm{DAB}(0.02 \%), \mathrm{H}_{2} \mathrm{O}_{2}(0.005 \%)$ in $0.05 \mathrm{M}$ Tris buffer $(\mathrm{pH} 7.6)$ for 10 to $20 \mathrm{~min}$. The sections were washed with distilled water, air-dried, and mounted on a glass slide with Entellan.

The anti-AVP was kindly provided by Drs. ZIMMERMAN and NILAVER (Columbia Univ.) and anti-OXT was purchased from UCB Bioproducts, Belgium.

\section{Specificity check}

In order to test the specificity of the HRP reaction, the sections from rats without an HRP injection was processed in the same way as above. For the specificity check of the immunohistochemical staining, anti-AVP or anti-OXT was pre-incubated with 1 to 10 $\mu \mathrm{g}$ of AVP or OXT for $1 \mathrm{~h}$ at $37^{\circ} \mathrm{C}$ prior to their use in immunohistochemical staining.

\section{RESULTS}

\section{The injection site}

The black HRP reaction product was seen throughout the posterior pituitary. In addition, moderate staining was seen in the pars intermedia and that portion of the pars distalis which faced the pituitary cleft. When the HRP was injected outside the posterior pituitary, no reaction product was observed in the hypothalamus or extrahypothalamic area.

Fig. 1. A-F. Double-stained neurons in the rat hypothalamus. Black dots in the cytoplasm indicate the HRP retrogradely transported from the posterior pituitary. The brown diffuse staining is the DAB reaction product of immunocytochemical staining. The asterisks in A, B, C, F indicate the third ventricle. A. PVN stained with anti-AVP. A majority of the neurons in the lateral part of this nucleus were labeled with HRP and most of them were also stained with AVP. B. PVN stained with anti-OXT. Many neurons double-stained with HRP and OXT are scattered in the ventro-lateral region of this nucleus. Note that there are some neurons in the dorso-medial region which are stained with anti-OXT but not with HRP (open arrow). C. A part of the anterior commissural nucleus at a high magnification. A neuronal perikarya (thick arrow), which is double-stained with HRP and anti-OXT, is situated in the ependymal cell layer. A nerve fiber is seen to project toward the ventricular lumen (thin arrow). Arrowheads indicate the surface of the ependyma facing the ventricular lumen. D. SON stained with anti-AVP. A majority of the neurons were labeled with HRP. There are many neurons in the ventral part of this nucleus which are stained both with HRP and anti-AVP. E. A part of PVN stained with anti-AVP. Three neurons (thin arrows) are labeled with both retrograde HRP and anti-AVP, and some others are labeled with only HRP (arrowheads). One neuron (thick arrow) is stained only with anti-AVP. F. The most caudal part of PVN. The neurons near the third ventricle are stained with only anti-OXT, while at the lateral area (open arrow) there are some neurons which are labeled with HRP and/or immunoreactions by anti-OXT. A and B: $\times 130, \mathrm{C}: \times 800, \mathrm{D}: \times 210, \mathrm{E}: \times 760, \mathrm{~F}: \times 120$. 
A
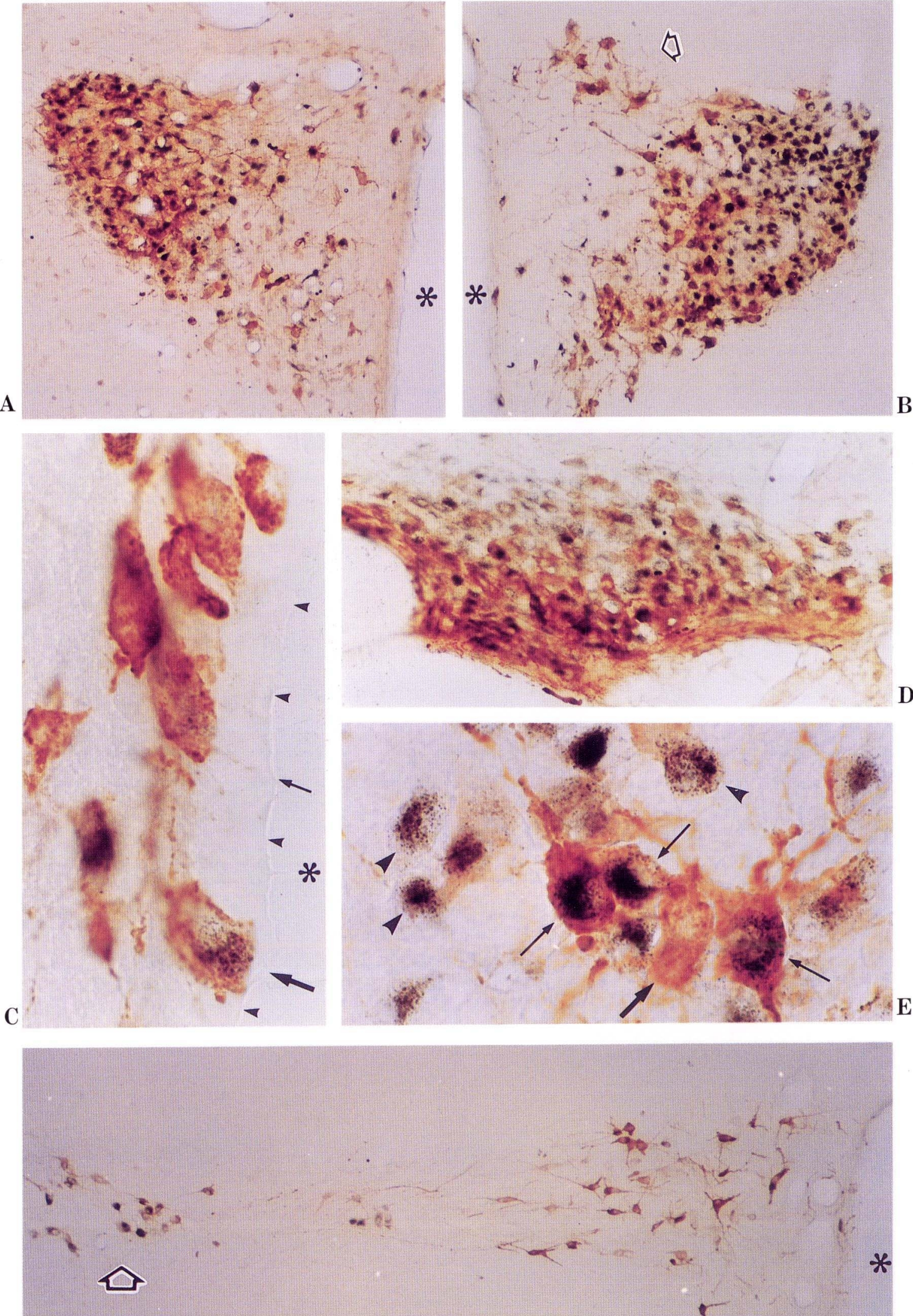
86 Y. TANigUCHI et al.:
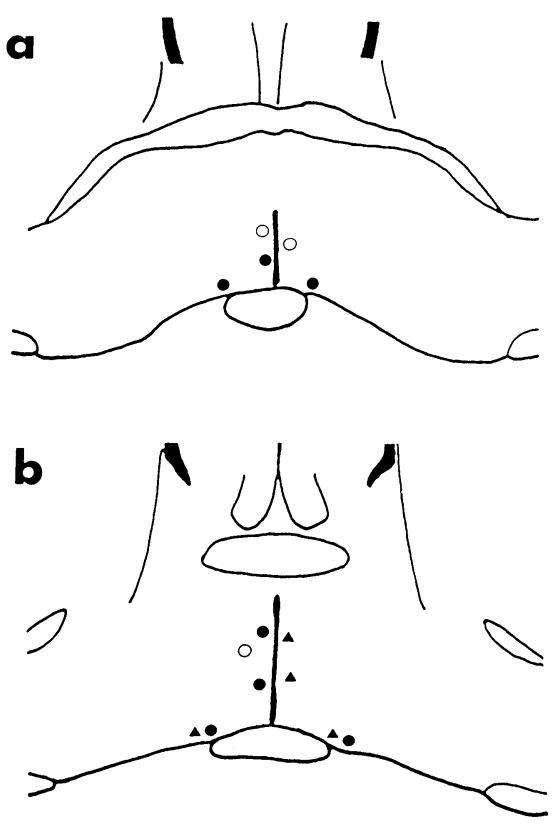

C

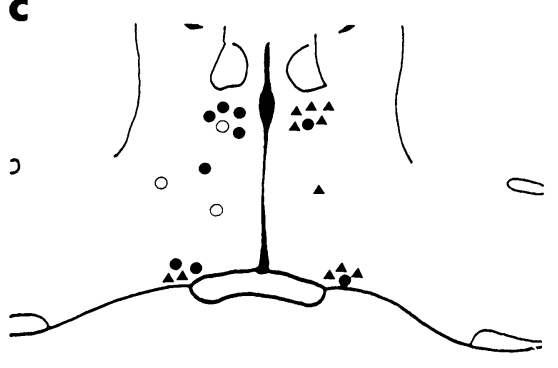

d
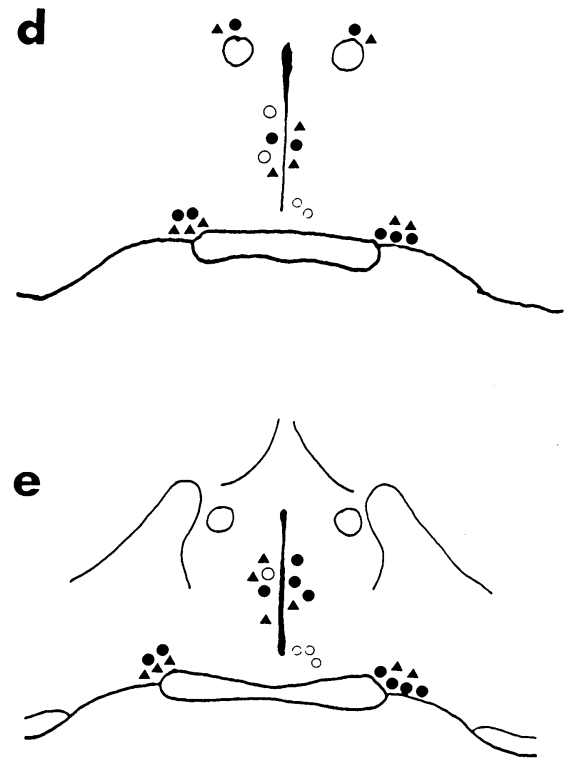

f

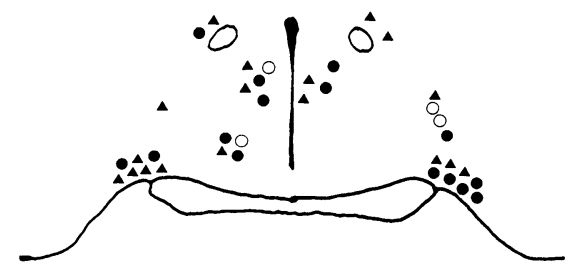

g
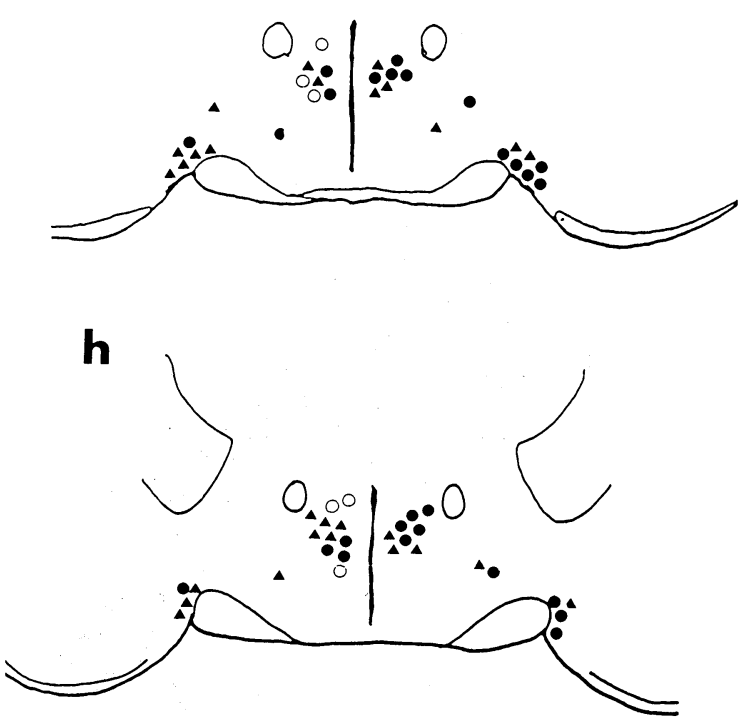

i
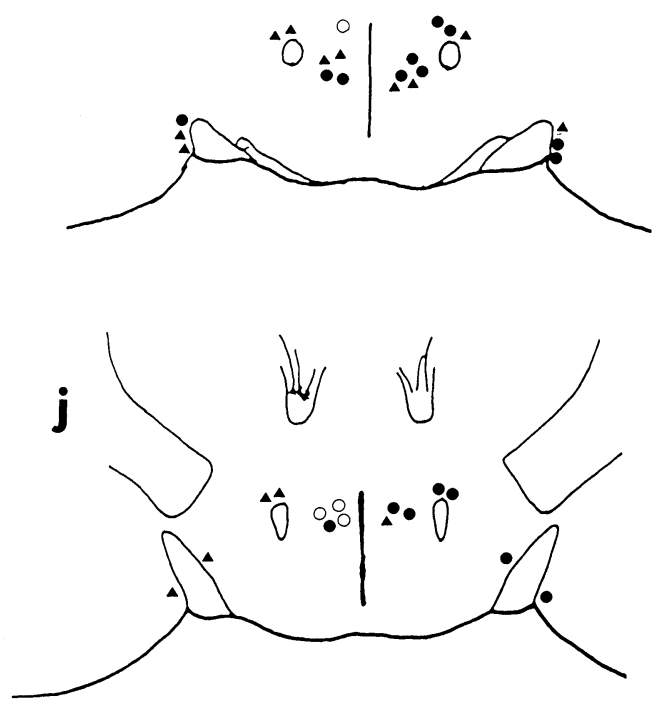

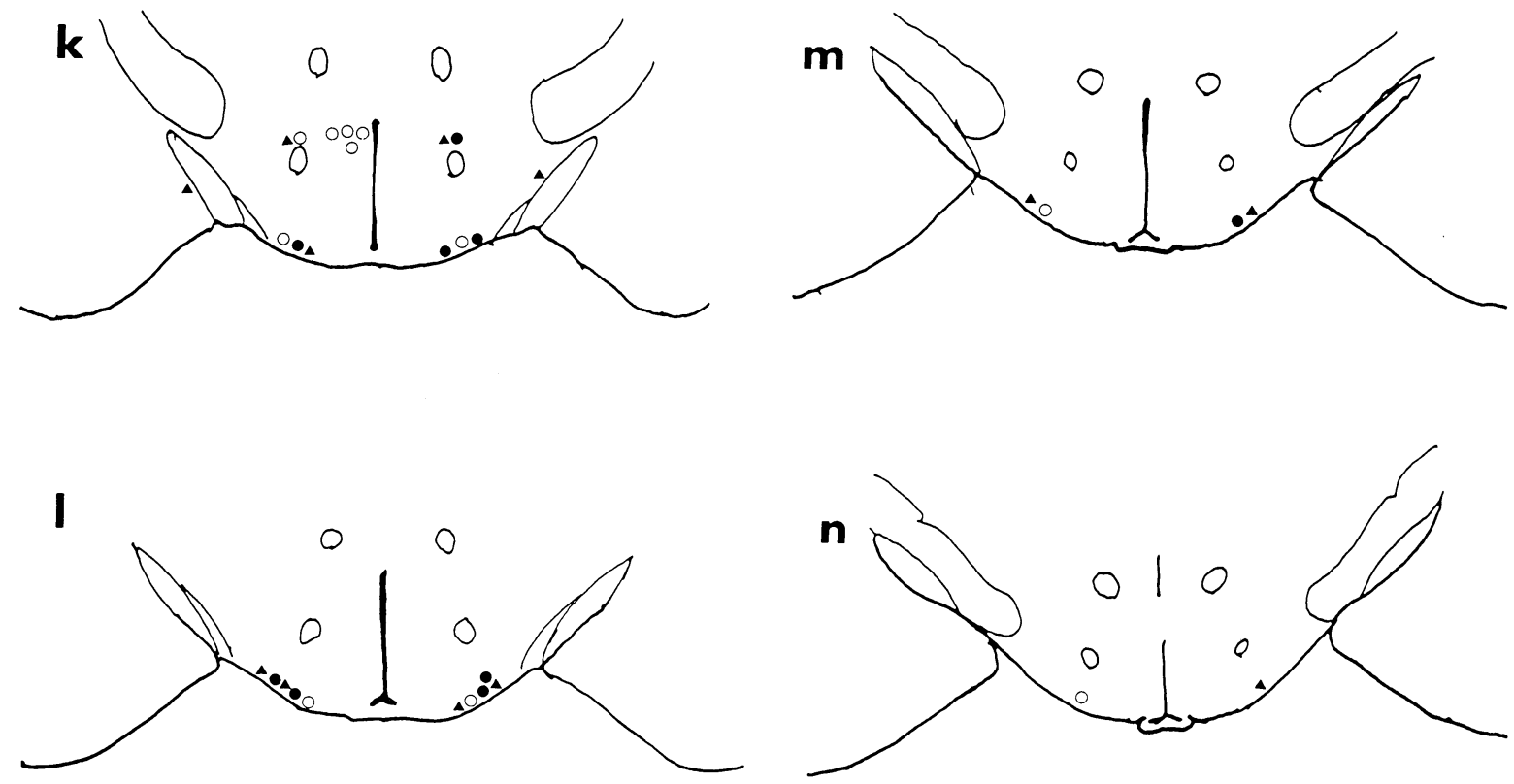

Fig. 2. A diagrammatic representation of perikarya labeled with HRP (closed triangles), immunostained with anti-AVP or anti-OXT (open circles), or double-stained with both HRP and the neuropeptide (closed circles). The left half of each figure depicts the results for OXT-neurons, and the right half for AVP-neurons. a-n represent the cross sections of the rat brain arranged in rostro-caudal order according to the atlas by KÖNIG and KLIPPEL (1963). The distance (in $\mu \mathrm{m}$ ) from the interaural line: a 7190, b 6860, c 6670, d 6360, e 6060, f 5910, g 5780, h 5660, i 5340, j 5150, k 4840, l 4380, m 4110, n 3750.

\section{The distribution of HRP-labeled and/or immuno- cytochemically stained neurons}

The reaction product of HRP and the immunocytochemical staining of the neuropeptide was easily discriminated in the double-stained sections as shown in Figure 1C, E. The HRP reaction product was seen as black dots in the cytoplasm of the neuronal perikarya, while the immunocytochemical staining was presented as diffuse brown staining in the perikarya and processes. Some neurons were labeled only with black dots, some were labeled only with brown diffuse staining, while others were double-labeled with both of them. The distribution of the neurons labeled with HRP, anti-AVP or anti-OXT is summarized in Figure 2.

In the supraoptic nucleus (SON), a majority of the neurons were double stained with HRP and the neuropeptide (AVP or OXT), but a small number of neurons were stained only with the neuropeptide (Figs. $1 \mathrm{D}, 2 \mathrm{c}-\mathrm{g})$. The retrochiasmatic part of SON consisted of a mixture of neurons stained only with HRP, AVP or OXT, and neurons double-labeled with HRP and the neuropeptide (Fig. 2k-m).

In the paraventricular nucleus (PVN), nearly all the neurons in the lateral magnocellular region were double-stained with HRP and the neuropeptide (Figs. 1A, B, 2f-k).

There were, however, two exceptions. First, a number of neurons in the dorso-medial portion of this nucleus were stained only with OXT (Figs. 1B, 2h). Second, neurons at the posterior end of this nucleus were also stained only with OXT without showing retrogradely transported HRP (Figs. 1F, 2k).

In the suprachiasmatic nucleus (Fig. $2 \mathrm{~d}$, e), virtually no neuron was recognized to be labeled with HRP throughout this study (we could find only one labeled neuron in this nucleus), though in its vicinity one or two neurons were occasionally seen to be labeled with HRP.

The other magnocellular nuclei of the hypothalamus, i.e., the accessory magnocellular nuclei (Fig. 2fh) and neurons of the perifornical region (Fig. 2d, f, i, j) comprised four kinds of neurons: those stained only with HRP, AVP or OXT, and those double-stained with HRP and the neuropeptide. There were some of 
the neurons labeled with HRP in almost every magnocellular nucleus.

\section{CSF-contacting neurons}

In the course of this study we often observed that some of the perikarya labeled with HRP were situated just beneath the ependymal layer, or in certain cases, within the ependymal layer (Fig. 1C). These neurons, which are known as 'cerebrospinal fluid $(C S F)$ contacting neurons', were often additionally stained with anti-OXT (Fig. 1C). In some rare cases they were stained with anti-AVP. Also, OXT- or AVP-containing fibers were frequently found within the ependymal layer. The perikarya and fibers of these CSF-contacting neurons were observed from the plane of the anterior commissural nucleus to the caudal end of the PVN. Sometimes perikarya in the subependymal layer were observed sending processes to the ventricular lumen (Fig. 1C).

\section{DISCUSSION}

The specificity of the HRP reaction was proved by the fact that no reaction product was observed in the neuronal perikarya of the hypothalamus-including the PVN and $\mathrm{SON}$ - when the sections from the rats without HRP injection were processed in the same way as the experimental ones. The specificity of the immunostaining was confirmed by the preabsorption test as described above. The anti-AVP and anti-OXT sera had a weak cross reaction with OXT and AVP, respectively, but this was deemed to have no bearing on this study because the specific staining was much stronger than the cross reaction and was distinguished clearly.

As shown in Figure 1C, E, the black HRP reaction product was clearly observed in the neuronal perikarya with or without immunohistochemical staining, showing a diffuse brown coloring. In regard to the interference of the HRP reaction with the immunostaining, there is a point which should be reported in detail. When the sections were immunostained after incubation in the DAB-cobalt solution, the immunohistochemical reaction was supressed to a certain degree. This effect was conspicuous when the incubation time exceeded $10 \mathrm{~min}$. We therefore employed two modifications to overcome this problem. First, the colchicine injection was given one day before sacrifice to facilitate the immunostaining of the neuronal perikarya. We also tried to reduce the concentration of the cobalt chloride, finding that $0.02 \%$ was sufficient to make the HRP reaction product uniformly black. By these modifications it became possible to ensure that AVP or OXT were immunostained with only a slight decrease in staining intensity even after the detection of HRP in the DABcobalt solution. In addition, the animals remained in good condition even after surgery, and the HRP seemed to be effectively taken up by the neurons. As a result, an incubation time of less than 5 min was usually required for the detection of HRP.

In the pituitary, the HRP reaction product was observed not only in the pars nervosa but also in the pars intermedia and that part of the pars distalis which faced the pituitary cleft. This was due to the diffusion of the HRP solution during the insertion and drawing out of the micropipette and was unavoidable with the injection method emlpoyed. However, the undesirable diffusion into the pars intermedia or pars distalis can be discounted, because when the injection was done outside of the posterior pituitary, no HRP. labeled neuron was observed in the brain regions studied.

In general, the distribution of HRP-labeled neurons was similar to that already reported by others (SCHERLOCK et al., 1975; ONO et al., 1978; HOSOYA and MATSUSHITA, 1979; ARMSTRONG et al., 1980; KELly and SwANSON, 1980; SwANSON and KUYPERS, 1980).

As has been already reported by some investigators, magnocellular neurons can be classified into two categories according to the destination of their fiber projection: those projecting to the posterior pituitary and those projecting to brain stem or spinal cord. In the PVN, the dorso-medial region and the most caudal region have been reported to project to the brain stem or spinal cord, with the other regions to the posterior pituitary (ONO et al., 1978; HosOYA and MATSUShitA, 1979; ARMSTRONG et al., 1980; SwAN SON and KUYPERS, 1980). As a whole, our results were consistent with these reports, and the fact that different regions of PVN project to different parts of the brain was further confirmed by a direct visualization of retrogradely transported HRP and the neuropeptides in the same tissue section.

In the course of this study, we often observed the 'CSF-contacting neurons,' which were reported in mammals to be situated in the subependymal layer, with their cytoplasmic processes projecting to the ventricular lumen (VIGH and Vigh-TeICHMAnN, 1973). During this study, however, we often found HRP-labeled neuronal perikarya not only beneath the ependymal layer but also within the ependymal layer. The presence of these CSF-contacting, and OXT- or AVP-containing neurons suggests that some of the neurons 
which project to the posterior pituitary have a direct connection with the cerebrospinal fluid. This is interesting in view of the fact that the posterior pituitary is involved in the regulation of the osmolarity of body fluids. It may be possible that some neurons contacting CSF and at the same time projecting to the posterior pituitary receive information concerning the osmolarity of the cerebrospinal fluid directly from the ventricular lumen.

\section{REFERENCES}

Adams J. C.: Technical considerations on the use of horseradish peroxidase as a neuronal marker. Neuroscience 2: 141-145 (1977).

Armstrong, W. E., S. Warach, G. I. Hatton and T. H. MCNEILL: Subnuclei in the rat hypothalamic paraventricular nucleus: a cytoarchitectural, horseradish peroxidase and immunocytochemical analysis. Neuroscience 5: 1931-1958 (1980).

Beitz, A. J., J. R. Clements, M. A. Mullet and L. J. ECKLUND: Differential origin of brainstem serotonergic projections to the periaqueductal gray and superior $\mathrm{col}_{-}$ liculus of the rat. J. Comp. Neurol. 250: 498-509 (1986).

Hancock, M. B.: Cells of origin of hypothalamo-spinal projections in the rat. Neurosci. Lett. 3: 179-184 (1976).

Hosoya, Y. and M. Matsushita: Identification and distribution of the spinal and hypophyseal projection neurons in the paraventricular nucleus of the rat. A light and electron microscopic study with the horseradish peroxidase method. Exp. Brain Res. 35: 315-331 (1979).

KeLLY, J. and L. W. Swanson: Additional forebrain regions projecting to the posterior pituitary: preoptic region, bed nucleus of the stria terminalis, and zona incerta. Brain Res. 197: 1-9 (1980).

KöNIG, J. F. R. and R. A. KLIPPEL: The rat brain: A stereotaxic atlas of the forebrain and lower parts of the brain stem. Wiliams and Wilkins Co., Baltimore, 1963.

Mikami, S., H. Tokado and D. S. Farner: The hypothalamic neurosecretory systems of the Japanese quail as revealed by retrograde transport of horseradish peroxidase. Cell Tiss. Res. 194: 1-15 (1979).

Ono, T., H. Nishino, K. Sasaka, K. Muramoto, I. Yano and A. Simpson: Paraventricular nucleus connections to spinal cord and pituitary. Neurosci. Lett. 10:141-146 (1978).

Peterson, R. P.: Magnocellular neurosecretory centers in the rat hypothalamus. J. Comp. Neurol. 128: 181-190 (1966).

Rhodes, C. H., J. I. Morrell and D. W. Pfaff: Immunohistochemical analysis of magnocellular elements in rat hypothalamus: distribution and numbers of cells containing neurophysin, oxytocin, and vasopressin. J. Comp. Neurol. 198: 45-64 (1981).
SaPer, C. B., A. D. Loewy, L. W. Swanson and W. M. Cowan: Direct hypothalamo-autonomic connections. Brain Res. 117: 305-312 (1976).

Sawchenko, P. E. and L. W. Swanson: Immunohistochemical identification of neurons in the paraventricular nucleus of the hypothalamus that project to the medulla or to the spinal cord in the rat. J. Comp. Neurol. 205: 260-272 (1982).

Scherlock, D. A., P. M. Field and G. Raisman : Retrograde transport of horseradish peroxidase in the magnocellular neurosecretory system of the rat. Brain Res. 88: 403-414 (1975).

Sofroniew, M. V. and U. Schrell: Hypothalamic neurons projecting to the rat caudal medulla oblongata, examined by immunoperoxidase staining of retrogradely transported horseradish peroxidase. Neurosci. Lett. 19: 257-263 (1980).

- : Evidence for a direct projection from oxytocin and vasopressin neurons in the hypothalamic paraventricular nucleus to the medulla oblongata: immunohistochemical visualization of both the horseradish peroxidase transported and the peptide produced by the neurons. Neurosci. Lett. 22: 211-217 (1981).

Stefanini, M., C. Martino and L. Zamboni: Fixation of ejaculated spermatozoa for electron microscopy. Nature 216: 173-174 (1967).

Swanson, L. W. and H. G. J. M. Kuypers: The paraventricular nucleus of the hypothalamus: cytoarchitectonic subdivisions and organization of projections to the pituitary, dorsal vagal complex, and spinal cord as demonstrated by retrograde fluorescence double-labeling methods. J. Comp. Neurol. 194: 555-570 (1980).

Swanson, L. W., P. E. Sawchenko, S. J. Wiegand and J. L. PRICE: Separate neurons in the paraventricular nucleus project to the median eminence and to the medulla or spinal cord. Brain Res. 198: 190-195 (1980).

Vigh, B. and I. Vigh-Teichman: Comparative ultrastructure of the cerebrospinal fluid-contacting neurons. Int. Rev. Cytol. 35: 189-251 (1973).

W AINER, B. H. and D. B. RYE: Retrograde horseradish peroxidase tracing combined with localization of choline acetyl-transferase immunoreactivity. J. Histochem. Cytochem. 32: 439-443 (1984).

\author{
Dr. Yutaka TANIGUCHI \\ Department of Morphology \\ Institute of Endocrinology \\ Gunma University \\ 3-39-15 Showamachi, Maebashi \\ 371 Japan \\ 谷口豊 \\ 371 前橋市昭和町 3-39-15 \\ 群馬大学内分泌研究所 \\ 形態学研究部
}

